\title{
Grain Boundary Properties and Grain Growth: Al Foils, Al Films
}

\author{
Katayun Barmak, Wayne E. Archibald, Anthony D. Rollett \\ Department of Materials Science and Eng., Carnegie Mellon University, Pittsburgh, PA 15213 \\ Shlomo Ta'asan, David Kinderlehrer \\ Department of Mathematical Sciences, Carnegie Mellon University, Pittsburgh, PA 15213
}

\begin{abstract}
Relative grain boundary energy as a function of misorientation angle has been measured in cube-oriented, i.e., $<100>$ fiber-textured, $120 \mu \mathrm{m}$-thick $\mathrm{Al}$ foil using orientation imaging microscopy and a statistical multiscale method. The energies of low-angle boundaries increase with misorientation angle, in good agreement with the Read-Shockley model. The relative energies of high-angle boundaries exhibit little variation with misorientation. Examination of the grain structure of $<111>$ fiber-textured, $100 \mathrm{~nm}$-thick Al films annealed at $400{ }^{\circ} \mathrm{C}$ for $0.5-10 \mathrm{~h}$ shows 5 and 6 sided grains to be the most frequent, and the fraction of four-sided grains to be significant. The mean number of sides is slightly lower than the expected value of 6 for twodimensional structures. Of lognormal, gamma and Rayleigh distributions, gamma gives the best fit to the grain size data in the films; however, the difference between gamma and lognormal is small. Grain growth is not self-similar and stagnates after one hour of annealing. The evolution of the grain size distribution with time indicates that the growth stagnation in the films is neither consistent with boundary pinning by grooving nor with conventional treatments of solute drag. Surface, elastic-strain and plastic-strain energy driving forces do not play a significant role in the grain growth and the subsequent stagnation since the films are strongly textured even in the asdeposited state. The steady-state distributions of reduced grain area for two-dimensional, Monte Carlo and partial differential equation based simulations show excellent agreement with each other, even when anisotropic boundary energies are used. However, comparison with experimental distributions reveals a significantly higher population of small grains in the experiments.
\end{abstract}

\section{INTRODUCTION}

To tailor the grain structure of materials, it is of interest to develop predictive models of grain growth that incorporate experimentally determined boundary properties and are validated against experimentally characterized grain structures. To this end, we have made use of cubeoriented $120 \mu \mathrm{m}$-thick foils of $\mathrm{Al}$ and electron back scatter diffraction in combination with a statistical multiscale method to determine the relative boundary energies of 890 boundaries as a function of misorientation angle. Grain growth has been examined in $100 \mathrm{~nm}$-thick films of Al using transmission electron microscopy. For each annealing time, the distribution of grain area, grain size, dihedral angle and the number of sides for between 737 and 2022 grains has been determined. The experimental grain structures are compared with those obtained in twodimensional (2D) simulations of grain growth that use both the Monte Carlo Potts (MC) model and the partial differential equation (PDE) model that tracks the curvature-driven evolution of the grain boundary network. 
As will be seen, the energies of low angle boundaries in the $\mathrm{Al}$ foil increase with misorientation angle, in good agreement with the Read-Shockley model, while those of highangle boundaries show little variation with misorientation. In the $\mathrm{Al}$ films, grain growth proceeds until approximately three out of four grains have disappeared, at which point the growth stagnates. Comparison of experiments and simulations shows a higher population of small grains in the experimental structures.

\section{EXPERIMENT AND SIMULATION}

The $\mathrm{Al}$ foils were $120 \mu \mathrm{m}$ thick and were taken from capacitor foil stock supplied by the Alcoa Corporation. Foil composition in weight \% was $99.98 \mathrm{Al}, 0.001 \mathrm{Si}, 0.002 \mathrm{Fe}, 0.006 \mathrm{~K}$, and $0.005 \mathrm{Cu} .1 \mathrm{~cm} \times 1 \mathrm{~cm}$ pieces of the foil were annealed in $\mathrm{Ar}-4 \% \mathrm{H}_{2}$ at $550{ }^{\circ} \mathrm{C}$ for $9 \mathrm{~h}$ to obtain a columnar grain structure with a strong cube texture.[1] The oxide layer present on the surface prevented thermal grooves from forming at the intersections of grain boundaries with the surface and thus enabled grain growth to occur to grain sizes larger than the foil thickness. The samples were air cooled following annealing and electropolished at room temperature for $60 \mathrm{~s}$ at $20 \mathrm{~V}$ in a solution of $730 \mathrm{cc}$ ethanol, $100 \mathrm{cc}$ ethylene glycol monobutylether, $78 \mathrm{cc}$ perchloric acid and $90 \mathrm{cc}$ distilled water.

Electron back scatter diffraction patterns and orientation image maps were obtained using a Philips XL-40 field emission gun scanning electron microscope/orientation imaging microscope (OIM). The scans were performed at $15 \mathrm{kV}$ with the sample tilted at $70^{\circ}$. The scan area was $500 \mu \mathrm{m} \times 500 \mu \mathrm{m}$, with a step size of $2 \mu \mathrm{m}$. Grain orientations and misorientations were obtained using the TSL software on the OIM. An example inverse pole figure map, more commonly known as an orientation image map, is shown in Fig. 1. The dihedral angles at grain boundary triple junctions, i.e., the angle between each pair of boundaries meeting at the triple point, were obtained from skeletonized boundary traces of the orientation image maps using the Linefollow code developed by Mahadevan.[2]
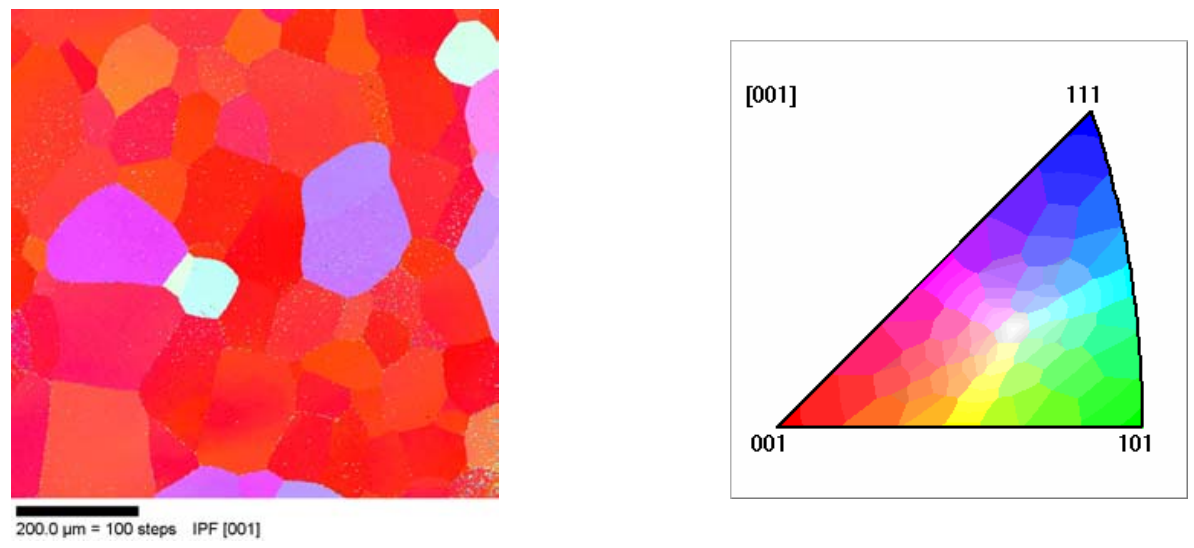

Fig. 1 - An inverse pole figure (IPF) map, also known as an orientation image map, of a 120 $\mu \mathrm{m}$-thick $\mathrm{Al}$ foil annealed for $9 \mathrm{~h}$ at $550{ }^{\circ} \mathrm{C}$, showing strong $<001>$ fiber-texture. 
The dihedral angle distribution is given in Fig. 2a. Relative grain boundary energies as a function of misorientation angle were obtained using the technique previously used by Yang et al. [1] to determine boundary energies in $\mathrm{Al}$ foils similar to those studied here. In this technique, triple junctions are assumed to be at equilibrium and to obey the Herring equation of tangential and normal (torque) force balance.[3] To simplify the analysis, it is further assumed that the grain boundary energy is independent of boundary orientation, i.e., the torque term is taken to be zero. Each pair of boundaries then obeys equations of the form $\sigma_{1} \sin \chi_{2}-\sigma_{2} \sin \chi_{1}=0$, known as Young's relations, where the subscripts denote the boundary (here boundary type as defined below) and the associated dihedral angle. The resultant system of linear equations for all the triple junctions in the dataset is solved using a statistical multiscale method, described in detail elsewhere. [4-7] For the present studies, the grain boundary misorientations were divided into thirteen types in the range of 0 to 60 degrees, noting that the maximum misorientation angle is limited to $62.8^{\circ}$ by symmetry.[8] The thirteen boundary types are listed in Table I and the misorientation angle distribution is given in Fig. $2 \mathrm{~b}$. With 13 types of boundaries, the maximum number of boundary pairings satisfying Young's relations is 78. For the present data set, 18 pairs were absent, resulting in only 60 pairs. The number of boundaries analyzed was 890 .

It should be noted that the foil was freestanding and not attached to a substrate, which tends to minimize internal stresses. In addition, since the foils developed a strong cube texture as a result of the annealing, which subsequently changed very little with further annealing, the crystallographic orientations of the planes at the surface of the foil were similar, and, as a result, the driving forces due to differences in surface energy were minimal in the above experiments. The primary driving force for boundary motion was that of curvature of the grain boundaries themselves. Also, the similarity in grain orientation tends to minimize differences in strain energy from residual stresses in the foil.

The 100-nm thick Al films were deposited at a rate of $0.13 \mathrm{~nm} / \mathrm{s}$ by DC magnetron sputtering from $99.99 \%$ pure targets. The total metallic impurity content of the target was 5.58 ppm by weight, with Fe accounting for $3.00 \mathrm{ppm}$ of this total. The substrates for film deposition were thermally oxidized 3 -inch diameter $\operatorname{Si}(100)$ wafers. The oxide thickness was $100 \mathrm{~nm}$. The substrates were rotated at rate of 10-15 rpm to ensure greater film thickness uniformity. The
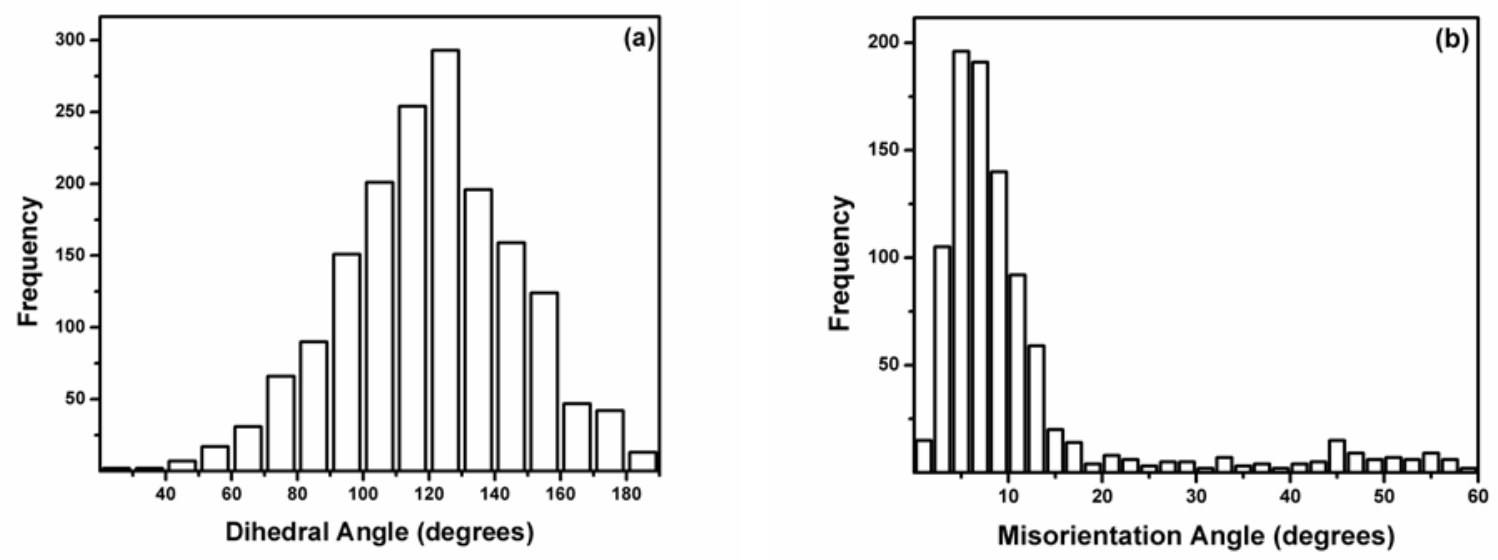

Fig. 2 - (a) Dihedral angle and (b) misorientation angle distributions for the Al foil in Fig. 1. 
Table I - Grain boundaries in $\mathrm{Al}$ foil sorted by misorientation angle.

\begin{tabular}{|c|c|c|c|}
\hline $\begin{array}{c}\text { Boundary } \\
\text { Type }\end{array}$ & $\begin{array}{c}\text { Misorientation Angle } \\
\text { (Degrees) }\end{array}$ & $\begin{array}{c}\text { Boundary } \\
\text { Type }\end{array}$ & $\begin{array}{c}\text { Misorientation Angle } \\
\text { (Degrees) }\end{array}$ \\
\hline 1 & $1-4$ & 8 & $26.1-34$ \\
\hline 2 & $4.1-6$ & 9 & $34.1-42$ \\
\hline 3 & $6.1-8$ & 10 & $42.1-46$ \\
\hline 4 & $8.1-10$ & 11 & $46.1-50$ \\
\hline 5 & $10.1-15$ & 12 & $50.1-54$ \\
\hline 6 & $15.1-18$ & 13 & $54.1-60$ \\
\hline 7 & $18.1-26$ & & \\
\hline
\end{tabular}

standard deviation in film thickness was found to be less than $2.2 \%$. The base pressure of the sputtering chamber was in the $10^{-8}$ Torr range. The sputtering gas was ultrahigh purity $\mathrm{Ar}$ $(99.9995 \%)$ at a pressure of $3 \mathrm{mTorr}$ and a flow rate of $20 \mathrm{sccm}$. The sputtering gas passed through an additional purifier before entering the chamber. Following deposition, the wafers were sectioned into $5 \mathrm{~mm} \times 5 \mathrm{~mm}$ pieces, and the pieces were annealed at $400{ }^{\circ} \mathrm{C}$ in $\mathrm{Ar}-4 \% \mathrm{H}_{2}$, flowing at a rate of $100 \mathrm{sccm}$, for a series of times ranging from $0.5 \mathrm{~h}$ to $10 \mathrm{~h}$. Separate measurements indicated that samples would reach the annealing temperature in $\sim 5$ minutes.

Film texture was characterized for the as-deposited and the $10 \mathrm{~h}$-annealed samples by measuring the $\{111\}$ and $\{200\}$ pole figures in $5^{\circ}$ steps in sample tilt and rotation angles in a Philips X'Pert System. The texture data is presented here as a $\{111\}$ fiber plot wherein the intensity of the $\{111\}$ reflection is averaged over the rotation angle and plotted against the sample tilt angle. Film grain structure was characterized in the transmission electron microscope. The electron transparent samples were obtained by chemically back-etching the silicon and stopping in the oxide layer.[9] The samples were examined in a Philips EM420 microscope operating at $120 \mathrm{kV}$. A $10 \mu \mathrm{m}$-diameter objective aperture was used to maximize the diffraction contrast, as shown in Fig. 3. The samples were imaged at a magnification of 60,000, except for some samples that were additionally imaged at a magnification of 36,000 or 49,000 . For the same field of view, two to four bright-field images were recorded at different sample tilts to make the grain boundaries more easily identifiable (Fig. 3). The tilt angle was kept small in order to minimize the effect of foreshortening. The grain boundaries were traced by hand from enlarged photographic prints. Each tracing was examined by a second observer, and, in cases where the position of a boundary was in question, the grain boundary and the affected neighbors were omitted from subsequent analysis. The black-on-clear tracings of the grain boundaries were digitally scanned into image files (Fig. 4), and the grain area and size, the latter defined as the diameter of a circle of equal area to the grain of interest, was obtained using Scion image in a manner similar to our previous work. $[9,10]$ The dihedral angles were obtained using the Linefollow code mentioned above.[2] The number of grain sides, i.e., the number of nearest neighbors of a grain, were obtained by manual counting (Fig. 4).The number of grains in each sample for which the area was measured was in the range of 1100-2022. The number of grains for which the nearest neighbors were measured was smaller and in the range of 737-1089.

The experimental grain size distributions were fitted to three analytical distributions, namely the lognormal, gamma, and Rayleigh. The lognormal distribution is given as $f(x)=(1 / \beta x \sqrt{2 \pi}) \exp \left[-(\ln x-\alpha)^{2} / 2 \beta^{2}\right]$, where $\alpha$ and $\beta$ are independent fitting parameters. 

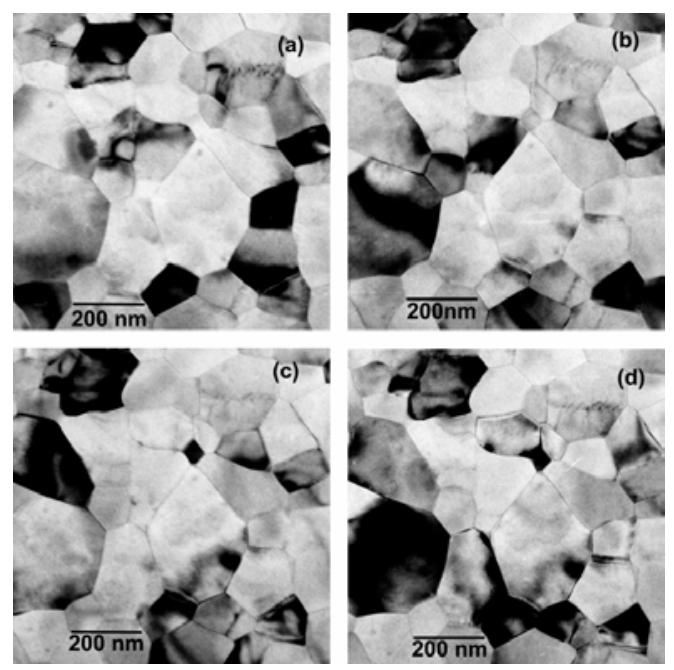

Fig. 3 - Bright-field transmission electron micrographs of a $100 \mathrm{~nm}$-thick $\mathrm{Al}$ film annealed at $400{ }^{\circ} \mathrm{C}$ for 2 hours taken at sample tilt angles of (a) 0 , (b) 0.5 (c) 0.75 and (d) $1^{\circ}$, respectively.

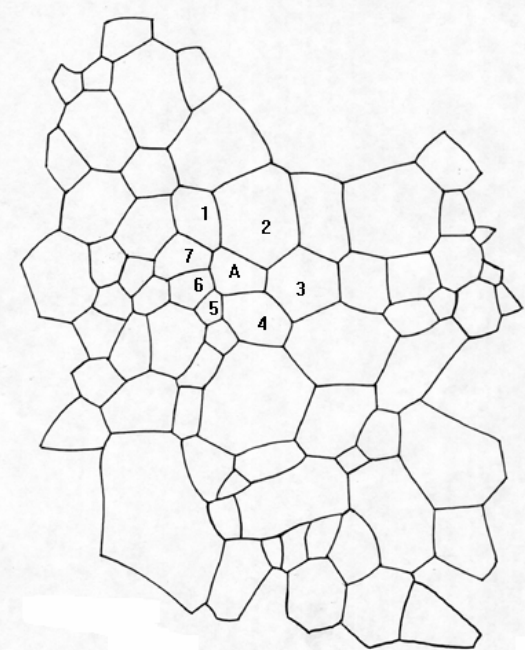

Fig. 4 - Hand-traced grain boundaries obtained from a series of bright-field transmission electron micrographs such as those shown in Fig. 3.

The arithmetic mean and the standard deviation of $\operatorname{lnx}$ are defined respectively as $\mu=\exp \left(\alpha+\beta^{2} / 2\right)$ and $\sigma=\exp \left(2 \alpha+\beta^{2}\right) \exp \left(\beta^{2}-1\right)$. The general form of the gamma distribution function is given as $f(x)=\left(1 / \beta^{\alpha} \Gamma(\alpha)\right) x^{\alpha-1} \exp (-x / \beta)$, where $\alpha$ and $\beta$ are the median and logarithm of the standard deviation, respectively, and $\Gamma(\alpha)=\int_{0}^{\infty} x^{\alpha-1} \exp (-x) d x$.[1113] The Rayleigh distribution is given as $f(x)=(\alpha x / \beta) \exp \left(-x^{2} / 4 \beta\right)$, where $\alpha$ and $\beta$ are constants.[14] These three functions were fitted to the data, using the maximum likelihood estimate, and the Kolmogorov-Smirnov (K-S) test was used to determine the goodness of fit.[11]

For grain growth simulations both the Monte Carlo Potts (MC) model and a boundary tracking model termed the partial differential equation (PDE) model were used.[15-18] The latter simulations portray the evolution of a network of two-dimensional curves governed by the Mullins Equation of curvature driven growth with the Herring Condition of force balance imposed at triple junctions. The Herring Condition is, in fact, the natural boundary condition for equilibrium of the stationary Mullins Equation. The system of partial differential equations is dissipative and, importantly, the algorithmic implementation is also dissipative. Critical events which result in change of network topology such as grain disappearance and edge swapping or disappearance have been incorporated using a semi-discrete method that conserves the dissipative character of the system. The simulation methodology allows the use of both isotropic and anisotropic grain boundary energies (and mobilities). For simplicity, the anisotropic energy used here has the form $\sigma=1+0.12 \sin ^{2} \theta$, where $\theta$ is the misorientation angle. As noted above, the energy was reconstructed from experimental data in the $\mathrm{Al}$ foil sample ignoring the influence of the torque terms which occur in the Herring Condition. This procedure identifies the shape of the energy, but, as a rule of thumb, the energy must be scaled by a factor of 0.1 to represent the 
actual relative energy. The simulations discussed here are thus consistent with the experimentally actual relative energy. A typical computation begins with 25,000 grains and runs until about 8000 grains remain. Self-similarity of the relative area histograms occurs approximately one-half to two-thirds through the process, and the relative area histogram is found to be an extremely stable statistic of the simulation. Since grain growth or coarsening is a large-scale metastable process, there are no benchmark problems for testing the theory or its implementation. Instead, the simulations are verified with particular diagnostics, for example, average grain area grows linearly with time, and, for the case of isotropic boundary energy, the Mullins - von Neumann $(n-6)$ rule holds at the level of individual grains, and not merely on average. For additional information the reader is referred to [16] and, for a discussion and comparison with other methods to [17].

\section{RESULTS AND DISCUSSION}

Grain boundary energies in the $120 \mu \mathrm{m}$-thick Al foil determined based on dihedral angle measurements (Fig. 2b) showed good agreement to previous results of Yang et al. [1], as seen in Fig. 5. It should be noted that the variation in energy with misorientation yields relative energies only; therefore the new results presented here were multiplied by a constant chosen so as to maximize the agreement in the low angle range where the two datasets overlapped. Figure 5 further shows that for low-angle boundaries, the boundary energy has a reasonable fit to the Read-Shockley dislocation model for low-angle boundaries, namely $E=E_{o} \theta(A-\ln \theta)$, where $E$ is the boundary energy, $E_{o}$ is a function of the elastic properties of the material, $\theta$ is the misorientation angle across the grain boundary, and $A$ is a constant that depends on the dislocation core energy. [19] For high-angle boundaries, as found by Yang [20] and others [21,

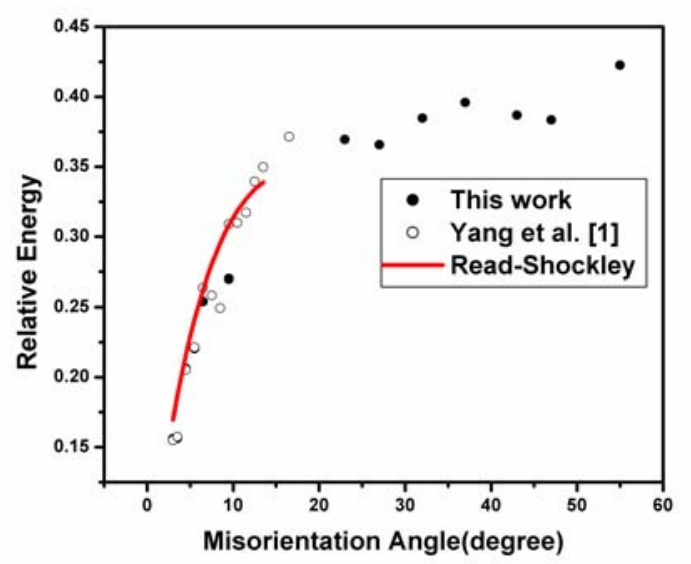

Fig. 5 - Relative grain boundary energy as a function of misorientation angle for the Al foil in Fig. 1. 
22], the variation in grain boundary energy with misorientation is not large (Fig. 5). The only exception to this statement is for coherent twin boundaries $\left(60^{\circ}\right.$ about $<111>$ in pure twist configuration) and $\sum 11$ boundaries with a (113) normal (i.e., a symmetric tilt configuration). Neither of these special boundary types is distinguishable in this (relatively small) dataset.

Characterization of the grain structure of the as-deposited and $400{ }^{\circ} \mathrm{C}$-annealed, $100 \mathrm{~nm}$ thick Al films showed the minimum number of sides to be 3 for all the samples, and the maximum number to lie in the range of 11-14 (Fig. 6). The most frequently observed numbers of nearest neighbors or sides were 5 and 6 . The mean number of sides for all the samples was either 5.8 or 5.9, compared with the expected value of 6 for interior polygons.[23] The reason for the lower experimental values is not the presence of quadruple junctions since care was taken to eliminate such junctions from the tracings. Instead, it may be a consequence of the presence of edge grains in the tracings. As can be seen in Fig. 4, these tracings are only finite, rather than infinite, arrays of polygons.[23] The dihedral angles at triple junctions were found to have a minimum in the 20 's and a maximum of $180^{\circ}$, reflecting the anisotropy of grain boundary energy. The average dihedral angle at triple junctions was $120^{\circ}$, as might be expected for twodimensional boundary networks meeting at triple points.

As an example, Fig. 7 shows the grain size data for the $2 \mathrm{~h}$-annealed, $100 \mathrm{~nm}$-thick Al film fitted with all three distributions, namely lognormal, gamma and Rayleigh. The K-S test showed that, with the exception of the $4 \mathrm{~h}$-annealed sample, the gamma distribution gave a better fit to the grain size data (Fig. 7). This observation contrasts with the work of Carpenter et al. where the lognormal distribution showed a slightly better fit to the experimental grain size data for 8185 grains in a thin $\mathrm{Al}$ film, and to other works where the grain size data is said to have a lognormal distribution. $[12,13,24]$ However, it is worth noting that the difference between the lognormal and gamma distributions is not large.

The grain size distributions for the Al films in Fig. 8, plotted as a fraction of grains of a

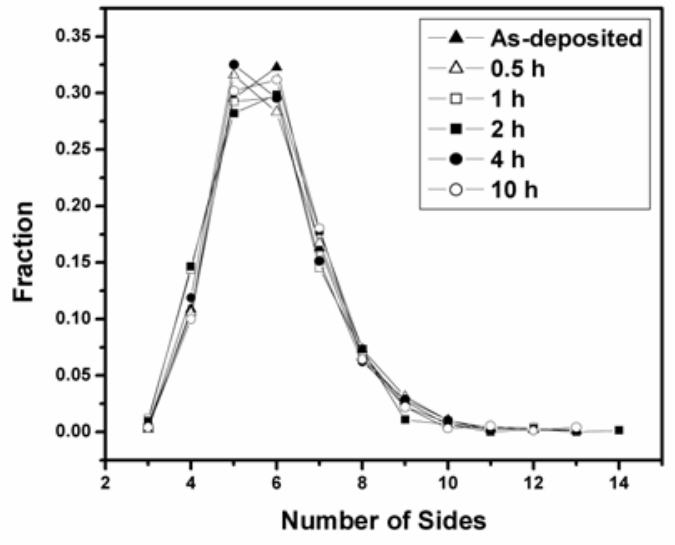

Fig. 6 - Number of sides distributions for as-deposited and $400{ }^{\circ} \mathrm{C}$ - annealed $100 \mathrm{~nm}$-thick Al films.

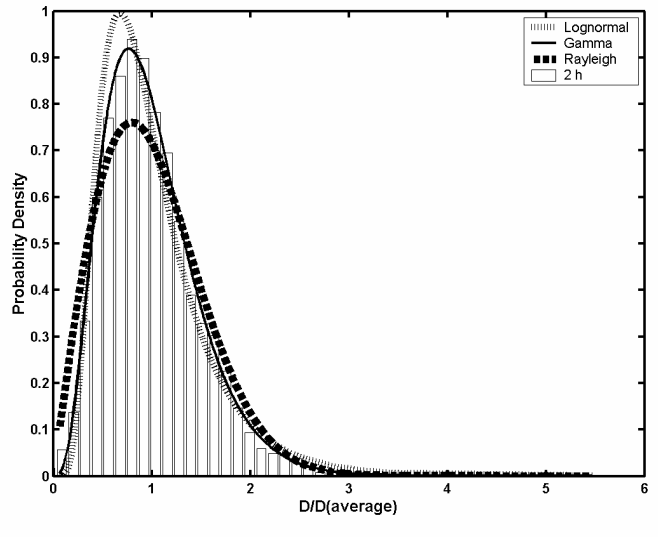

Fig. 7 - Grain size distribution for a $100 \mathrm{~nm}$ thick Al film annealed at $400{ }^{\circ} \mathrm{C}$ for 2 hours fitted to lognormal, gamma and Rayleigh distributions. 


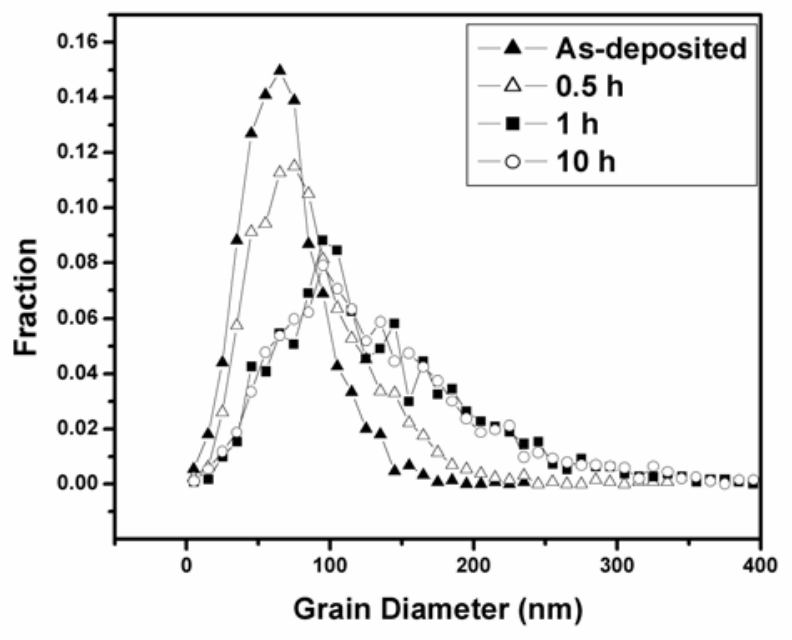

Fig. 8 - Fraction of grains versus grain size, defined as equivalent circular diameter, for as-deposited and $400^{\circ} \mathrm{C}$ - annealed $100 \mathrm{~nm}$-thick $\mathrm{Al}$ films.

given size versus size to account for the differing number of grains measured in each sample, evidence grain growth with annealing until stagnation at $1 \mathrm{~h}$. The stagnation is clearly seen in the overlap of the $1 \mathrm{~h}$ and $10 \mathrm{~h}$ distributions and further noted in the behavior of the mean grain size and standard deviation in Figs. 9a and b. The grain diameter approximately doubles from 68 $\mathrm{nm}$ for the as-deposited state to 134-145 $\mathrm{nm}$ for the stagnant structure. Figures 8 and $9 \mathrm{~b}$ also show that the distributions are not self-similar since instead of a rigid translation along the horizontal size axis, the distribution broadens with annealing. Furthermore, the distributions remain pinned at the smallest measured diameter (of 5-9 $\mathrm{nm}$ ).

Examination of the population of grains of a given size with annealing time up to the point of stagnation indicates an increase in the fraction of small grains. This increase is evidenced by, for example, the increasing separation of the mean and the median of the grain size from 4.7 to 8.5 to $10.6 \%$ for the as-deposited, the $0.5 \mathrm{~h}$-annealed and the stagnant structure, respectively. Note that the median is found be consistently smaller than the mean and that the stagnant state is taken as the combined statistics of 5902 grains for the 1, 2, 4 and 10-h annealed samples. Thus, it appears that the growth of grains and the subsequent stagnation are associated with an accumulation of small grains, an interpretation of which is that the small grains are not shrinking as fast as they should to allow the distribution to remain self-similar. This interpretation is also consistent with pinning of the distributions at the low end shown in Fig. 8.

Stagnation of grain growth has been previously attributed to pinning of grain boundaries by grooves formed at the intersection of the boundary with the film surface. $[25,26]$ However, given that $\mathrm{Al}$ forms an oxide upon exposure to air that is extremely stable, grooves are not expected to form in our films. [27] Furthermore, grooving should pin the boundaries with low curvature, whereas our data indicates that it is the boundaries of small grains, which by necessity have high curvature, that are the affected boundaries. A similar argument would apply to solute drag as a source of boundary pinning, since again the low curvature boundaries should be the affected boundaries.[24] In addition, the impurity content of the films is low given the high 

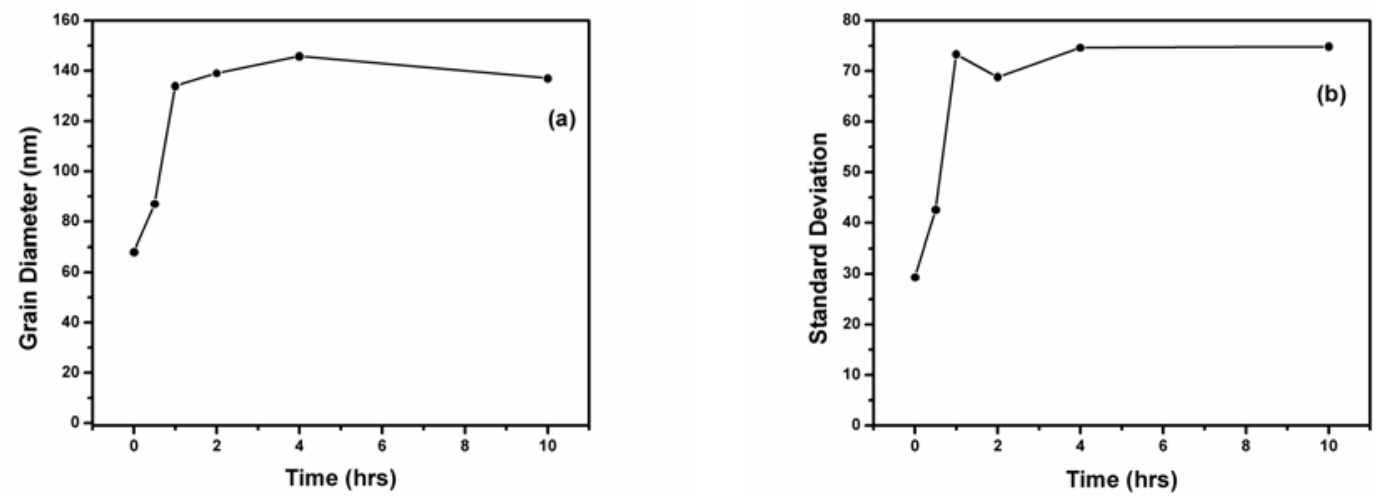

Fig. 9 - (a) Mean and (b) standard deviation of grain size as a function of time for $100 \mathrm{~nm}$-thick $\mathrm{Al}$ films annealed at $400^{\circ} \mathrm{C}$.

purity of the sputtering target and that of the sputtering gas. For the given target purity, the average Fe content of the boundaries for the as-deposited film is calculated as only 1 atom in $10^{4}$, or 20 atoms per boundary, assuming that all the Fe atoms are to be found in the boundaries of a columnar structure and the boundaries to have a width of $0.5 \mathrm{~nm}$.

It is worth noting that in thin films driving forces other than grain boundary energy reduction can be operative. Examples include surface, elastic-strain and plastic-strain energies. The minimization of these energies favors the growth of certain subpopulation of grains, and leads to the development of strong film texture.[28] However, for the films studied here, the minimization of these energies is not expected to play a significant role in either the initial grain growth or the eventual stagnation since the films were very strongly $<111>$-fiber textured even in the as-deposited condition and annealing resulted in minimal strengthening of this texture, as seen in the fiber plot of Fig 10. In addition, the Al films are in the zero stress, or lowcompressive steady-state stress state at the annealing temperature and reach this state during heating to temperature.[29-31] Thus, film stress and its relaxation are also not expected to play a significant role in the observed grain growth followed by stagnation.

The steady-state distributions for the simulated structures are given in Fig. 11. As can be seen, independent of the choice of simulation methodology (MC or PDE), and independent of the use of isotropic or anisotropic boundary energies, the distributions show excellent agreement. There is also no stagnation in grain growth and the distributions are found to be self-similar.

Comparison of the simulated and experimental structures shows higher probabilities for small grains in the latter compared with the former, whether the grains are still growing $(0.5 \mathrm{~h})$ or whether they have reached the stagnant stage, as seen in Fig. 12. The conclusion that can be drawn is that grain growth in these $100 \mathrm{~nm}$-thick Al films is neither well-represented by the 2D Monte Carlo model nor is it 2D curvature-driven coarsening as embodied in the Mullins equation with the Herring boundary condition, irrespective of the anisotropy of the boundary energy. This is either a consequence of the finite thickness of the films, given that thin films are not strictly 2D systems, or it implies the need for the inclusion of other terms in the equations for grain boundary motion. 


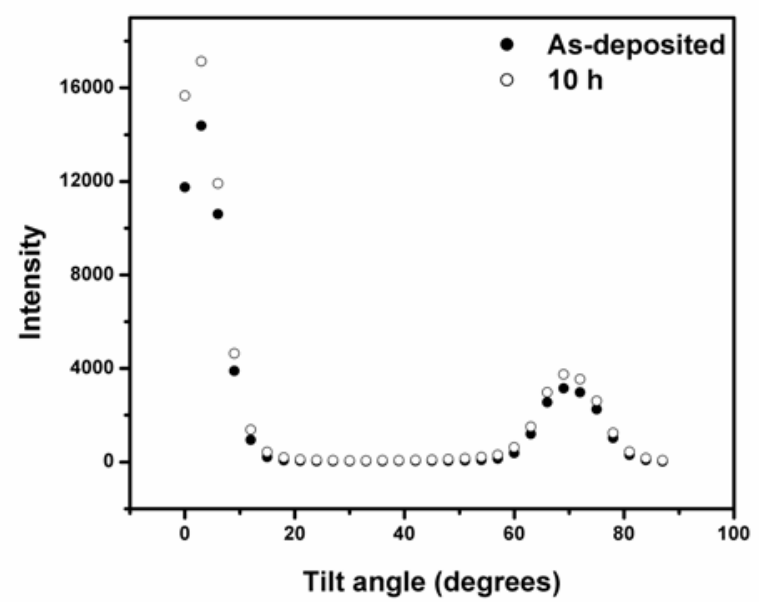

Fig. $10-<111>$ fiber plots for as-deposited and $400{ }^{\circ} \mathrm{C}, 10$ hourannealed 100 nm-thick Al film.

\section{CONCLUSIONS}

For the cube-oriented $120 \mu \mathrm{m}$-thick Al foil, the energies of low-angle boundaries increase with misorientation angle, in good agreement with the Read-Shockley model. For the high-angle boundaries there is little variation of energy with misorientation.

For the of $100 \mathrm{~nm}$-thick Al films, the number of sides is in the range of 3 to14, with a significant fraction of four-sided grains present in all the samples. With the exception of the $4 \mathrm{~h}-$ annealed sample, the grain size (diameter) distributions show a better fit to the gamma distribution when compared with the lognormal distribution; however, the difference between these two distributions is small.

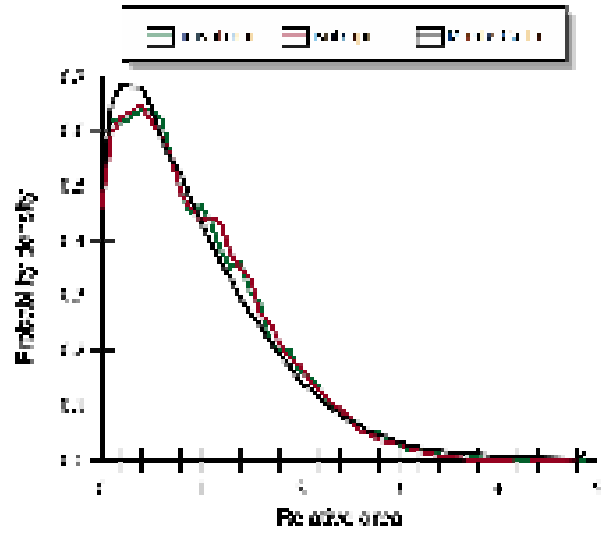

Fig. 11 - Steady-state probability densities versus reduced or relative area (area divided by average area) from simulations. 


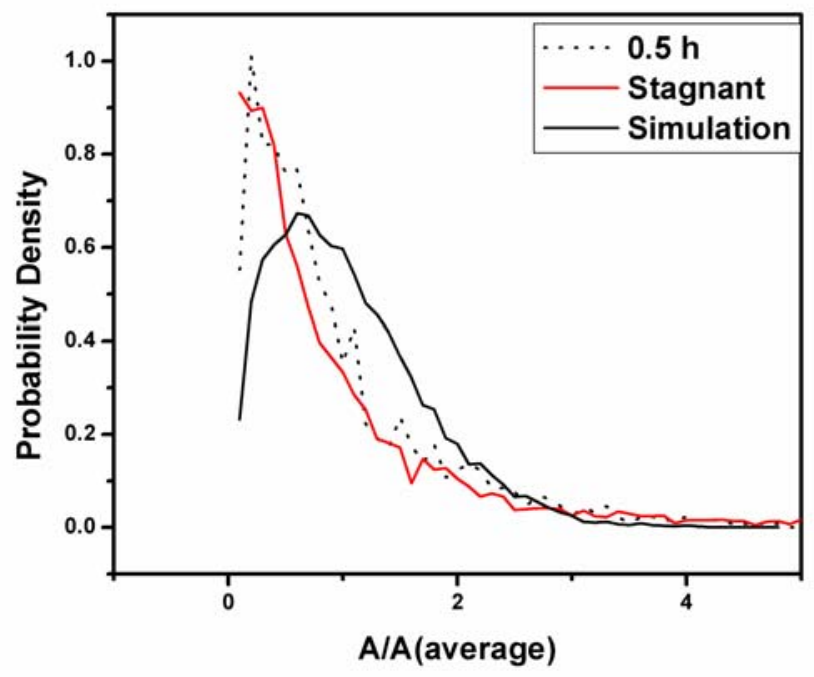

Fig. 12 - Comparison of reduced area probability densities for simulation and experiment.

Annealing the $100 \mathrm{~nm}$-thick films at $400{ }^{\circ} \mathrm{C}$ results in the growth of grains until stagnation is reached at $1 \mathrm{~h}$. Examination of the grain size distributions shows an increase in the population of small grains, indicating that the small grains are not disappearing as fast as they should. Grain growth stagnation in the films is neither consistent with boundary pinning by grooving nor with conventional treatments of solute drag, since it is the population of small grains that increases with annealing time. Further, since the Al films are very strongly $<111>$ fiber-textured even in the as-deposited state, there is no reason for surface, elastic-strain and plastic-strain energy driving forces to play a significant role in the grain growth and the subsequent stagnation.

Steady-state, reduced area distributions for MC and PDE simulations show excellent agreement with each other. However, comparison with experimental distributions evidences a significantly higher population of small grains in the experiments. The reason for the slow disappearance of small grains, the lack of self-similarity, and the eventual stagnation in the Al films remains unclear.

\section{ACKNOWLEDGMENTS}

Support from the MRSEC program of the National Science Foundation under award number DMR-0079996 is gratefully acknowledged. P. Yu and I. Livshits are thanked.

\section{REFERENCES}

1. C.C. Yang, W.W. Mullins, A.D. Rollett, Scripta Metall. 44, 2735 (2001). 
2. S. Mahadevan, D. Casasent, Proc. SPIE 4735, 104 (2002).

3. C. Herring, The Physics of Powder Metallurgy, ed. W. E. Kingston, (McGraw-Hill Book Co., New York 1951) p. 143.

4. B. L. Adams, D. Kinderlehrer, W. W. Mullins, A. D. Rollett, and S. Ta'asan, Scripta Mater., 38, 531 (1997).

5. D. Kinderlehrer, I. Livshits, S. Ta'asan, and D. E. Mason, Proc. ICOTOM-12, ed. J. A. Szpunar, NRC Research Press, 1643 (1999).

6. B. L. Adams, D. Kinderlehrer, I. Livshits, S. Ta'asan, D. E. Mason, W. W. Mullins, G. S. Rohrer, A. D. Rollett, D. M. Saylor, and C.-T. Wu, Int. Sci. 7, 321 (1999).

7. D. Kinderlehrer, I. Livshits, D. Mason, S. Ta'asan, Interface Sci. 10, 232 (2002).

8. F.C. Frank, Met. Trans. 19A, 403 (1988).

9. J. Kim, Ph.D. Thesis, Lehigh University, Bethelehem (2001).

10. http://www.scioncorp.com

11. W. Mendenhall, R.L. Scheaffer, D.D. Wackerly, Mathematical Statistics with Applications

(PWS Publishers, Boston, MA 1986) pp. 141-145, pp. 381-438.

12. D. T. Carpenter, J. M. Rickman, K. Barmak, J. Appl. Phys. 84, 5843 (1998).

13. D. T. Carpenter, J. R. Codner, K. Barmak, J. M. Rickman, Mater. Lett. 41, 296 (1999).

14. N. P. Louat, Acta Metall. 22, 721 (1974).

15. D. Srolovitz, J. Vac. Sci. Technol. A4, 2925 (1986).

16. D. Kinderlehrer, I. Livshits, S. Ta'asan, unpublished.

17. S. Ta'asan, P. Yu, I. Livshits, D. Kinderlehrer, J. Lee, Proc. $44^{\text {th }}$ AIAA/ASME/ ASCE/AHS

Structures, Structural Dynamics and Materials, Norfolk, VA, in press.

18. D. Kinderlehrer, I. Livshits, F. Manolache, A. D. Rollett, S. Ta'asan, Mater. Res. Soc. Symp. Proc. 652, Y1.5.1 (2001).

19. W. T. Read and W. Shockley, Phys. Rev. 78, 275 (1950).

20. C. C. Yang. Ph.D. Thesis, Carnegie Mellon University, Pittsburgh (2000).

21. G. C. Hasson, C. Goux, Scripta Metall. 5, 889 (1971).

22. D. Wolf, J. Mater. Res. 5, 1708 (1990).

23. E. R. Underwood, Quantitative Stereology, (Addison Wesley, Reading, MA 1970) p. 218.

24. H. J. Frost, Y. Hayashi, C. V. Thompson, D. T. Walton, Mater. Res. Soc. Symp. Proc. 317, 431 (1994).

25. W.W. Mullins: Acta Metall., 6, 414 (1958).

26. H. J. Frost, C. V. Thompson, D. T. Walton, Acta Metall. Mater. 381455 (1990).

27. T. B. Read, Free Energy of Formation of Binary Compounds - An Atlas of Charts for HighTemperature Chemical Calculations, (The MIT Press, Cambridge, Massachusetts, 1971) p. 27. Equilibrium oxygen partial pressure for dissociation of aluminum oxide at $400{ }^{\circ} \mathrm{C}$ is $10^{-175} \mathrm{~atm}$.

28. C. V. Thompson, R. Carel, Mater. Sci. Eng. B32, 211 (1995).

29. Gerth, D. Katzer, M. Krohn, Thin Solid Films 208, 67 (1992).

30. M. Legros, K. J. Hemker, A. Gouldstone, S. Suresh, R.-M. Keller-Flaig, E. Arzt, Acta Mater. 50, 3435 (2002).

31. J. Koike, S. Utsunomiya, Y. Shimoyama, K. Maryuama, H. Oikawa, J. Mater. Res. 13, 3256 (1998). 